\title{
Femtosecond laser pulse induced desorption: a molecular dynamics simulation
}

\author{
Ivor Lončarića, ${ }^{\mathrm{a}, *}$, Maite Alducin ${ }^{\mathrm{a}, \mathrm{b}}$, Peter Saalfrank ${ }^{\mathrm{c}, \mathrm{b}}$, J. Iñaki Juaristi ${ }^{\mathrm{d}, \mathrm{a}, \mathrm{b}}$ \\ ${ }^{a}$ Centro de Física de Materiales CFM/MPC (CSIC-UPV/EHU), P. Manuel de Lardizabal 5, 20018 San Sebastián, Spain \\ ${ }^{b}$ Donostia International Physics Center DIPC, P. Manuel de Lardizabal 4, 20018 San Sebastián, Spain \\ ${ }^{c}$ Institut für Chemie, Universität Potsdam, Karl-Liebknecht-Strasse 24-25, D-14476 Potsdam, Germany \\ ${ }^{d}$ Departamento de Física de Materiales, Facultad de Químicas, Universidad del País Vasco (UPV/EHU), Apartado 1072, \\ 20080 San Sebastián, Spain
}

\begin{abstract}
In recent simulations of femtosecond laser induced desorption of molecular oxygen from the $\mathrm{Ag}(110)$ surface, it has been shown that depending on the properties (depth and electronic environment) of the well in which $\mathrm{O}_{2}$ is adsorbed, the desorption can be either induced dominantly by hot electrons or via excitations of phonons. In this work we explore whether the ratios between the desorption yields from different adsorption wells can be tuned by changing initial surface temperature and laser pulse properties. We show that the initial surface temperature is an important parameter, and that by using low initial surface temperatures the electronically mediated process can be favoured. In contrast, laser properties seem to have only a modest influence on the results.
\end{abstract}

Keywords: laser induced desorption, molecular dynamics with friction, local density friction approximation, generalized Langevin oscillator model

\section{Introduction}

Laser pulses can be used as an efficient way of promoting reactions at surfaces [1-4]. They can also induce reactions that can not be activated thermally [5]. Consequently, the theoretical modelling of such processes is of great interest. In this article we concentrate on the modelling of a prototypical reaction, desorption of a molecule from a metal surface, which is induced by an intense femtosecond laser pulse. Moreover, we consider only laser wavelengths that can not couple directly to the molecule, such that all processes are metal surface mediated (wavelengths in the UV-NIR region). The process of desorption under these conditions is usually called desorption induced by multiple electronic transitions (DIMET) [6]. Several experimental studies of DIMET (using different diatomic molecules and different metals) established that the

\footnotetext{
*Corresponding author

Email address: ivor.loncaric@gmail.com (Ivor Lončarić)
}

desorption yield $Y$ increases with the absorbed laser fluence $F$ as a power law, $Y=a F^{n}$, with exponent $n=2-10$ [1, 5, 7], 11].

As proposed in Ref. [12, femtosecond laser induced desorption can be modelled by the socalled molecular dynamics with electronic friction model [12 16]. In this model, the motion of the adsorbed molecule takes place in the ground state potential energy surface (PES) and the nonadiabatic coupling to the laser excited surface electronic system is included by friction and associated fluctuation forces. The friction forces account for the energy transfer from the adsorbate nuclear motion on the surface to the excitations of low energy electronhole pairs. The reverse process (electron-hole pairs deexcitations) results in fluctuation forces acting on the adsorbate that can be estimated from the frictional ones, via an effective electronic temperature. The effective electronic temperature is timedependent in response of the laser-pulse excitation and can be evaluated from a two-temperature model (2TM) [17, along with a phonon (substrate) 
temperature. This approach has the advantage that, due to its relative simplicity, it allows performing multidimensional dynamics simulations, in contrast to the models that explicitly include the excited electronic states $2 \sqrt{4}$.

To improve the accuracy and predictability over the empirically obtained PESs used in the first application of this method [12, 13], one can use density functional theory (DFT) to calculate the PES, as done by Luntz et al. 14. In that work, associative desorption of $\mathrm{H}_{2}$ from $\mathrm{Ru}(0001)$ was studied on the basis of a DFT-PES that included two molecular coordinates. A single surface coordinate was used in the dynamics to simulate laser heating of the surface. By using six-dimensional (6D) DFT-PES, this approach was extended to the full (6D) molecular dimensionality 15. However, the surface was kept frozen. Construction of a DFT-PES that includes also the surface atoms degrees of freedom is not computationally feasible. This presents a difficulty to model the laser excitation of surface phonons. To circumvent this problem we have recently proposed in Ref. 18 to couple the 6D molecular dynamics with electronic friction equations to the generalized Langevin oscillator (GLO) model [19 22] that describes the surface movement.

In the GLO model the surface is represented by a three dimensional (3D) harmonic oscillator that is coupled to a 3D ghost oscillator. The latter represents a thermal bath. In our laser induced desorption simulations, the temperature of this thermal bath is set such that it corresponds to the time evolution of the surface temperature that results from the application of the laser pulse according to the 2TM. The use of the GLO model enables us to keep the accuracy of the 6D DFT-PES and at the same time obtain a reasonable description of the phonon excitations.

We applied this model to simulate femtosecond laser induced desorption of $\mathrm{O}_{2}$ from $\mathrm{Ag}(110)$ [18. This system possesses several adsorption wells [23] as shown in Fig. 1 and, therefore, it is an interesting case study. The position and orientation of the molecule in the four adsorption wells are as follows: the center of mass $(\mathrm{CM})$ of the molecule at the long bridge site with the molecular axis oriented along the [001] direction (denoted LB), CM of the molecule at the short bridge site with the molecular axis oriented along the [1피 direction (denoted $\mathrm{SB}), \mathrm{CM}$ of the molecule at the hollow site with the molecular axis oriented along the [001] direction (denoted H001), and CM of the molecule at the
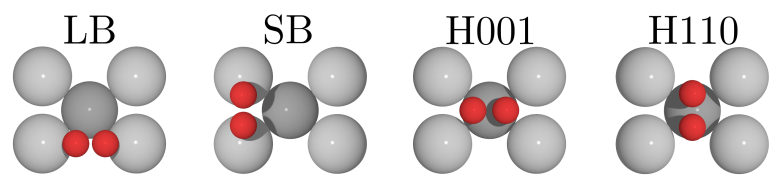

Figure 1: Positions of $\mathrm{O}_{2}$ in the four adsorption wells on $\mathrm{Ag}(110)$ as predicted by DFT calculations: long bridge (denoted LB), short bridge (denoted SB), hollow with molecular axis in [001] direction (denoted H001), and hollow with molecular axis in $[1 \overline{1} 0]$ direction (denoted H110). The $\mathrm{Ag}(110)$ surface is represented by light grey atoms for the first layer atoms and by dark grey atoms for the second layer atoms.

Table 1: Properties of the adsorption states of $\mathrm{O}_{2}$ on $\mathrm{Ag}(110)$ [18, 23]: Adsorption energy $E_{a}, \mathrm{O}_{2}$-surface distance $Z$, and electronic density in which oxygen atoms are embedded (expressed in terms of the mean free electron radius $r_{s}$, given in atomic units).

\begin{tabular}{cccc}
\hline Site & $E_{a}(\mathrm{eV})$ & $Z(\AA)$ & $r_{s}$ (a.u.) \\
\hline LB & -0.24 & 1.98 & 3.82 \\
SB & -0.33 & 2.20 & 3.57 \\
H001 & -0.24 & 1.29 & 2.62 \\
H110 & -0.21 & 1.09 & 2.57 \\
\hline
\end{tabular}

hollow site with the molecular axis oriented along the $[1 \overline{1} 0]$ direction (denoted H110). In all four adsorption wells, $\mathrm{O}_{2}$ is parallel to the surface, but with different distances from the surface as well as different adsorption energies, as shown in Table1. There is a clear difference between the bridge and the hollow wells regarding the distance of the well from the surface and, related to it, the surface electron density in which the molecule is embedded. The bridge wells are further away from the surface and the molecule is embedded in a lower electron density in comparison to the hollow wells. In Ref. [18, we studied the dependence of the laser induced desorption on these properties of the adsorption wells.

In general, we found that inclusion of phonon excitations in the desorption dynamics model is fundamental for this system. For the molecules adsorbed on the bridge sites, excitation of the surface phonons is the main desorption mechanism and the majority of desorption is happening well after the period in which there are large fluctuation forces due to the laser heated electrons. In contrast, for the molecules adsorbed in the hollow wells, the electronic mechanism dominates and most of the desorption is in the wake of the laser pulse. Interestingly, we found that the effect of the laser heated phonons is neither negligible in this case as they 
can take energy from the molecule and, thus, reduce the desorption probability. On the one hand, if we only account for phonon induced desorption, relative probabilities for desorption between the four wells can be connected to the adsorption well depth $E_{a}$. On the other hand, for the electronic mechanism, both $E_{a}$ and the electronic density in which the molecule is embedded are important.

Characteristic superlinear dependence of the desorption yield on the absorbed laser fluence, $Y=$ $a F^{n}$, was found from all four adsorption wells. Smallest desorption yields and largest exponent ( $n=5.8)$ are obtained for the SB well, while the largest desorption yields and smallest exponent $(n=2.6)$ are obtained for the H110 well, which is related to the differences in $E_{a}$ (see Table 1). Consistently larger desorption yields (as a function of fluence) are obtained from the H001 well in comparison to the LB well in spite of the fact that in these two wells $E_{a}$ is the same. This has been explained by the different electronic contributions to desorption for the two wells as discussed above.

In our previous study we made a specific choice of the laser pulse parameters and initial surface temperature [18. However, one could expect that the results may depend on these quantities. Here we explore their influence with the aim of understanding whether they can be used to tune the relative importance of electronic or phonon excitations. In this way, we could control the relative desorption yields from the different adsorption wells, by increasing desorption from some wells and reducing it from the others.

The article is organized as follows. The theoretical model is described in Sec. 2. Application of this model to study the influence of laser parameters and initial surface temperature to the desorption of $\mathrm{O}_{2}$ from $\mathrm{Ag}(110)$ is examined in Sec. 3 . The main conclusions of the article are summarized in Sec. 4

\section{Theoretical model}

A detailed description of the theoretical model and its implementation is given in Ref. [18. Briefly, we use the Langevin equation to model the dynamics of each atom $i$ in the molecule,

$$
\begin{aligned}
m \frac{d^{2} \mathbf{r}_{i}}{d t^{2}}= & -\nabla_{i} V\left(\mathbf{r}_{i}-\mathbf{r}_{s}, \mathbf{r}_{j}-\mathbf{r}_{s}\right) \\
& -\eta_{e l}\left(\mathbf{r}_{i}-\mathbf{r}_{s}\right) \frac{d \mathbf{r}_{i}}{d t} \\
& +\quad \mathbf{R}_{i}^{e l}, \quad i \neq j,
\end{aligned}
$$

where $m$ is the mass and $\mathbf{r}_{i}\left(\mathbf{r}_{j}\right)$ is the position vector of the oxygen atom $i(j)$. The first term in the right hand side (rhs) of Eq. (1) is the adiabatic force, which is here calculated using the $6 \mathrm{D}$ DFT-PES for $\mathrm{O}_{2}$ on $\mathrm{Ag}(110)$ obtained in Ref. 23 . Surface movement is described by a position vector $\mathbf{r}_{s}$ that varies with time according to the GLO model [19 22]. The GLO couples the $\mathrm{Ag}(110)$ surface to a thermal bath with temperature $T_{p h}(t)$ (see below). The second term in the rhs of Eq. (1) is the friction force proportional to the atom velocity and a position dependent friction coefficient $\eta_{e l}$. The latter is calculated using DFT for an atom embedded in a free electron gas with electron density equal to the surface electron density at the position of the atom (local density friction approximation) 24, 25. The third term in the rhs of Eq. (1) is the fluctuation force, calculated as a Gaussian white noise with variance $\operatorname{Var}\left(\mathbf{R}_{i}^{e l}\right)=\sqrt{2 k_{B} T_{e l}(t) \eta_{e l} / \Delta t}$, where $k_{B}$ is the Boltzmann constant, $\Delta t$ is the time integration step, and $T_{e l}(t)$ is the electronic temperature.

The electron, $T_{e l}$, and phonon, $T_{p h}$, temperatures are calculated in the so-called 2TM [17, which consists of two coupled diffusion equations,

$$
\begin{gathered}
C_{e l} \frac{\partial T_{e l}}{\partial t}=\frac{\partial}{\partial z} \kappa \frac{\partial T_{e l}}{\partial z}-g\left(T_{e l}-T_{p h}\right)+\frac{I(t) e^{-\alpha z}}{1-e^{-\alpha d}} \\
C_{p h} \frac{\partial T_{p h}}{\partial t}=g\left(T_{e l}-T_{p h}\right)
\end{gathered}
$$

where $C_{e l}$ is the electron heat capacity, $C_{p h}$ is the phonon heat capacity, $\kappa$ is the electron thermal conductivity, $g$ is the electron-phonon coupling constant, $\alpha^{-1}$ is the optical penetration depth, $d$ is the metal film thickness, and $I(t)$ is the adsorbed fraction of the laser pulse intensity. Only one spatial coordinate perpendicular to the surface $(z)$ is considered as typically laser spot sizes are relatively large. Solutions of Eqs. (2)-(3) for different initial surface temperatures $T_{i}=T_{e l}(0)=T_{p h}(0)$ and laser pulse durations, expressed as full width at half maximum (FWHM), are shown in Fig. 2

For each parameter set, the desorption dynamics results presented in Sec. 3 are obtained from 30000 trajectory calculations with an integration time of 50 ps. The molecule is initially in one of the adsorption wells, and when it reaches the distance of $6 \AA$ from the surface with positive velocity it is considered to be desorbed. We use Gaussian shaped pulses. Material constants and further details can be found in Ref. [18. 


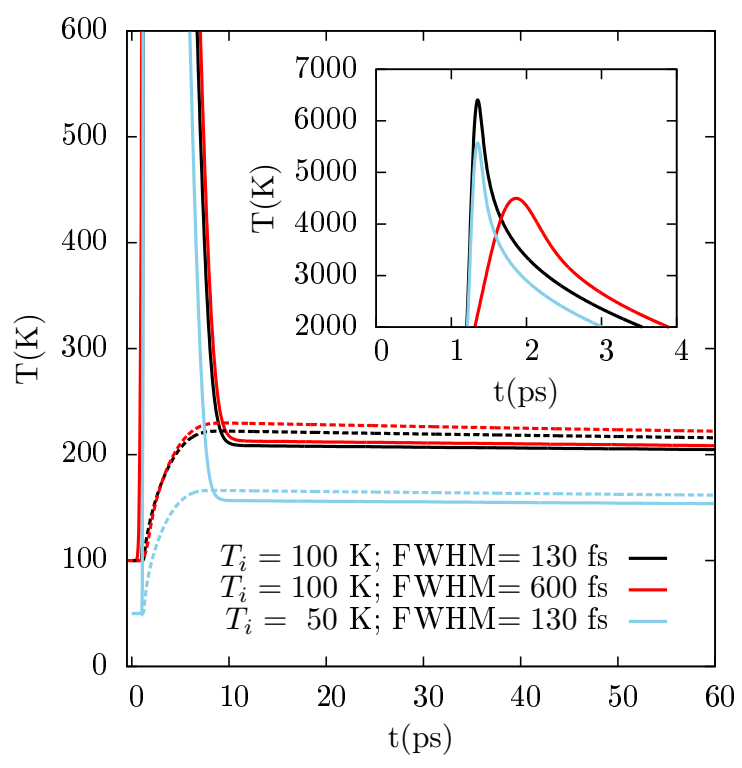

Figure 2: Electronic (solid line) and phonon (dashed line) temperatures as a function of time calculated within the 2TM for different initial surface temperatures $T_{i}$ and laser pulse widths (given as FWHM) with absorbed laser fluence $F=150 \mathrm{~J} / \mathrm{m}^{2}$. Inset: detailed view of the maxima reached by $T_{e l}$ in each case.

\section{Results}

When studying laser induced desorption, given a molecule/surface system, only the initial surface temperature and the laser pulse parameters remain to be chosen (at least in the model presented above). The laser pulse parameters are: fluence, pulse width, and wavelength. The dependence of laser induced desorption on laser fluence was already discussed above and in Ref. [18. Here we study the influence of the other quantities that one can control, starting with the initial surface temperature.

Solutions of the 2TM for two different initial surface temperatures, $T_{i}=50 \mathrm{~K}$ and $T_{i}=100 \mathrm{~K}$, are shown in Fig. 2. Clearly, the difference of $50 \mathrm{~K}$ in $T_{i}$ causes a similar difference of around $50 \mathrm{~K}$ in the equilibrated temperatures (both $T_{e l}$ and $T_{p h}$ ), which are achieved after $\sim 10$ ps. However, the maximum of $T_{e l}$, which is achieved in the wake of the laser pulse, is much reduced for the lower $T_{i}$ (see the inset in Fig. 2). A reduction of $T_{i}$ from $100 \mathrm{~K}$ to $50 \mathrm{~K}$ produces a reduction of the maximum of $T_{e l}$ that amounts to around $800 \mathrm{~K}$ (from $6300 \mathrm{~K}$ to $5500 \mathrm{~K}$ ). In the following, we study how this influences the desorption yield, which is defined by the

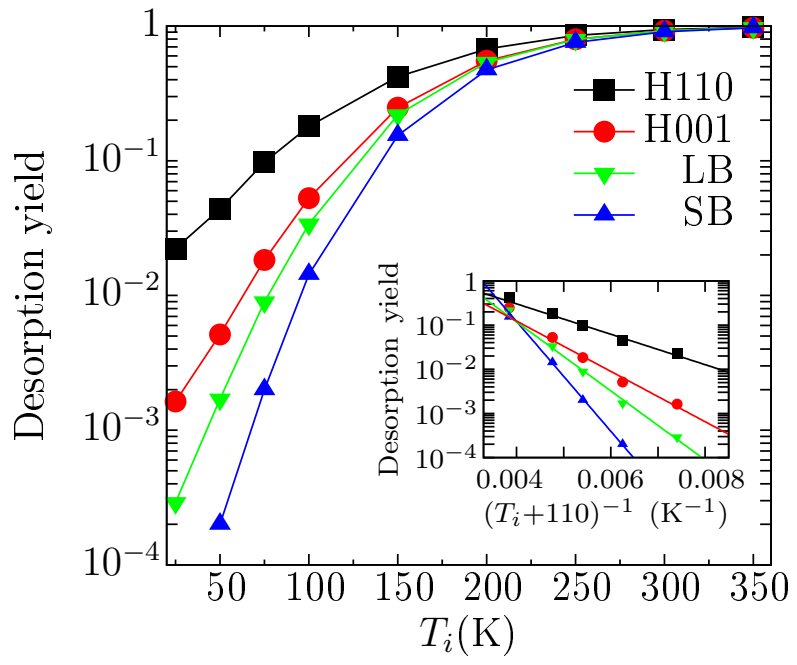

Figure 3: Desorption yield (ordinate in logarithmic scale) from the four wells (represented by different symbols and colors) as a function of the initial surface temperature $T_{i}$. In all cases FWHM $=130$ fs and $F=150 \mathrm{~J} / \mathrm{m}^{2}$. The inset shows the same data at $T_{i}<200 \mathrm{~K}$, plotted as a function of the inverse of the initial surface temperature $T_{i}$ increased by $110 \mathrm{~K}$ and a corresponding linear fit.

number of desorption events divided by the total number of trajectories.

Figure 3 shows that the desorption yield monotonically increases with increasing $T_{i}$. This is not surprising since, as we have already shown, increasing $T_{i}$ results in higher $T_{e l}$ and $T_{p h}$. For high initial surface temperatures, the desorption yield approaches 1 for all adsorption wells. The reason is that in the high initial surface temperature range, phonon temperatures are high enough to desorb all the molecules without any influence of the electronically mediated mechanism. At low $T_{i}$, one could try to understand the rapid increase of the desorption yield with $T_{i}$ using an Arrheniustype expression [15, 26. In the equilibrated regime $(t>10$ ps in Fig. 2), the molecule is expected to be well thermalized with the surface at temperature $T_{p h}$. Since for the short bridge well desorption is almost purely phonon mediated [18, the corresponding desorption rate at low $T_{i}$ can be approximated by the Arrhenius expression $\sim \exp \left[-E_{\mathrm{a}} / k_{B} T_{p h}(t)\right]$. As shown in Fig. 2, the equilibrated $T_{p h}$ is around $110 \mathrm{~K}$ higher than $T_{i}$ and it is almost constant with time $\left(T_{p h}\right.$ reduces only $3 \mathrm{~K}$ in the time period from 25 ps to $50 \mathrm{ps}$ due to the diffusion term). This allows us to write the Arrhenius expression as $\sim$ $\exp \left[-E_{\mathrm{a}} / k_{B}\left(T_{i}+110 \mathrm{~K}\right)\right]$. As this expression does 
not depend on time it is directly proportional to the desorption yield at low $T_{i}$. Indeed, the inset in Fig. 3 shows that the desorption yield increases exponentially with the negative inverse of $\left(T_{i}+110 \mathrm{~K}\right)$. We fit the data for desorption yield $Y$ with the expression $Y=b \exp \left[-E_{\text {eff }} / k_{B}\left(T_{i}+110 \mathrm{~K}\right)\right]$. Such a fit describes the data relatively good for all the wells with a very good fit obtained for the SB well. Fitting to the data for $T_{i}<100 \mathrm{~K}$ gives: $E_{\text {eff }}(\mathrm{SB})=$ $0.25 \mathrm{eV}, E_{\text {eff }}(\mathrm{LB})=0.16 \mathrm{eV}, E_{\text {eff }}(\mathrm{H} 001)=0.11 \mathrm{eV}$, and $E_{\text {eff }}(\mathrm{H} 110)=0.07 \mathrm{eV}$. The obtained values for $E_{\text {eff }}$ are reasonable in comparison to $E_{a}$ (see Table 1) for the bridge wells, especially for the SB well. Somewhat smaller $E_{\text {eff }}$ in comparison to $E_{a}$ is expected as in the dynamics most of the time molecules are not residing at the bottom of the well. For the hollow wells the poor agreement between $E_{\text {eff }}$ and $E_{a}$ is because $T_{e l}$ plays an important role for these wells. Since the dependence of $T_{e l}$ on $T_{i}$ is more involved it is not straightforward to explain the observed exponential dependence of the desorption yield on $T_{i}$ with the present simplified model.

It is interesting to look more closely at the H001 and LB wells, as they have the same adsorption energy but different dominant desorption mechanisms, electronic and phonon, respectively. Desorption from H001 is consistently higher. However, the ratio of the desorption yields is decreasing from $Y_{\mathrm{H} 001} / Y_{\mathrm{LB}}=3$ at $T_{i}=50 \mathrm{~K}$ to $Y_{\mathrm{H} 001} / Y_{\mathrm{LB}}=1$ at $T_{i} \geq 200 \mathrm{~K}$. This leads to the conclusion that if electronically mediated desorption is desired, it is necessary to have a low $T_{i}$.

The effect of the laser pulse FWHM on $T_{e l}$ and $T_{p h}$ is also shown in Fig. 2. In the long time regime, when the temperatures are equilibrated, we observe that $T_{e l}$ and $T_{p h}$ are only slightly modified by varying FWHM. In fact, when FWHM is increased from $130 \mathrm{fs}$ to $600 \mathrm{fs}$, the change in the equilibrated temperatures amounts to just $\approx 5 \mathrm{~K}$. This effect is, therefore, much less pronounced than the one we observed when varying $T_{i}$. However, we observe that when FWHM is increased from $130 \mathrm{fs}$ to $600 \mathrm{fs}$, the $T_{e l}$ curve broadens and the maximum value is reduced by $\approx 2000 \mathrm{~K}$.

The way these changes in $T_{e l}$ affect the desorption yields is shown in Fig. 4. As in previous cases, the largest desorption yields are obtained from $\mathrm{H} 110$, the well with the lowest adsorption energy, followed by H001, LB, and SB. There is a slight increase of the desorption yield values with increasing FWHM. In case of the bridge wells, for which desorption is dominated by phonons, this can

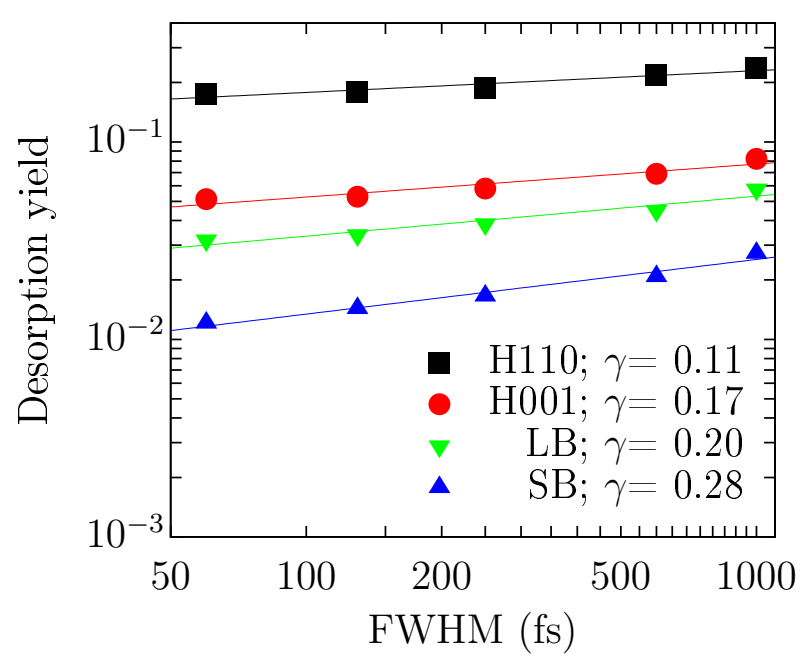

Figure 4: Desorption yield from the four wells (represented by different symbols and colors) as a function of the laser pulse FWHM (log-log scale). Data are fitted with a function $Y=c$ FWHM $^{\gamma}$. In all cases $T_{i}=100 \mathrm{~K}$ and $F=150 \mathrm{~J} / \mathrm{m}^{2}$.

be easily understood due to the small increase of the equilibrated $T_{p h}$ with FWHM. However, it is not straightforward to understand the observed behaviour in case of the hollow wells for which the electronic mechanism dominates, due to the more complicated behaviour of $T_{e l}$. On the one hand, since increase of FWHM results in somewhat larger equilibrated temperatures, it is expected that this increase has a positive contribution to the part of desorption induced by phonons, as in the case of the bridge wells. On the other hand, just from desorption yields, it is not clear what the contribution of the electronic channel is, as the peak maximum of $T_{e l}$ is considerably reduced, but the peak is broadened.

To gain a better insight on this issue we plot in Fig. 5 desorption rates as a function of time, for two different laser pulse FWHMs. For the bridge wells, as expected, the desorption rates increase with FWHM due to the larger phonon temperatures in the equilibrated regime. Interestingly, electron mediated desorption from the hollow wells in the wake of the laser pulse is also increased. This shows that in order to promote desorption via the electronic mechanism (under the studied conditions) larger desorption is obtained by increasing the time interval in which $T_{e l}$ is high than by increasing the maximum value of $T_{e l}$.

As shown in Fig. 4, the increase of the desorption 


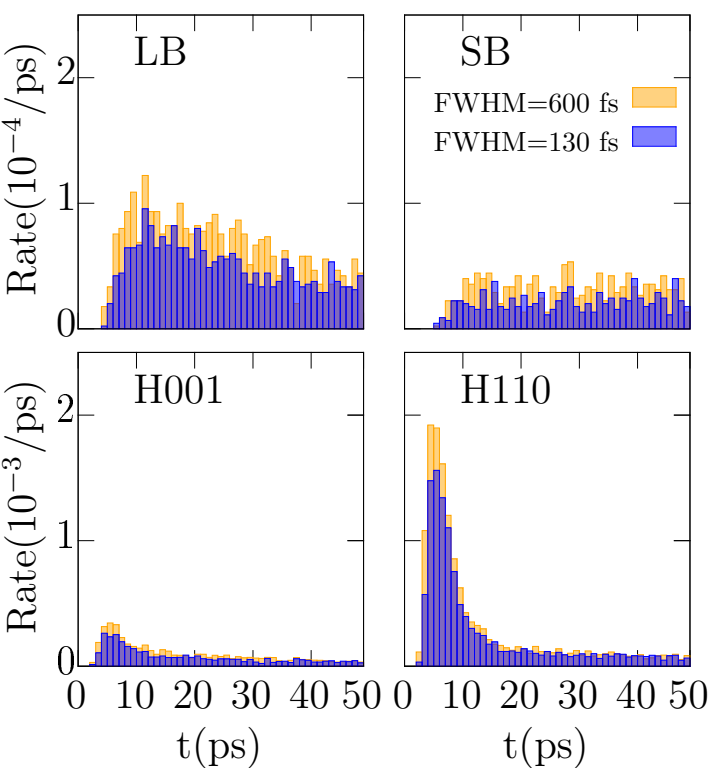

Figure 5: Desorption rates from the four wells for two different laser pulse widths, FWHM $=600$ fs (orange boxes) and FWHM $=130$ fs (blue boxes). Note the different scales in upper and lower panels. In all cases $F=150 \mathrm{~J} / \mathrm{m}^{2}$ and $T_{i}=100 \mathrm{~K}$.

yields with the laser pulse FWHM can be also fitted with a power law in which exponents are smaller than 1 . This contrasts with the power law that describes the dependence on laser fluence in which exponents are larger than 1 . The values of exponents range from $\gamma=0.11$ for the H110 well to $\gamma=0.28$ for the SB well, which approximately correspond to the 10-th and the 4-th root of FWHM, respectively. The ratio between desorption yields from different wells does not significantly depend on FWHM. This means, that tuning of FWHM can not be used to favour either electron or phonon mediated processes, at least in the range of FWHM $=60-1000 \mathrm{fs}$ that is explored here.

Finally, we discuss the effect of the laser wavelength $\lambda$, which enters Eq. (2) through the optical penetration depth $\alpha^{-1}=\lambda /(4 \pi k)$, where $k$ is the imaginary part of the refractive index (also called extinction coefficient) of the surface. For wavelengths $\lambda>360 \mathrm{~nm}$, the extinction coefficient $k$ of $\mathrm{Ag}$ increases linearly with $\lambda$ [27, 28, so that $\alpha^{-1}$ remains constant, resulting in the same $T_{e l}$ and $T_{p h}$, and consequently, the same desorption dynamics. All the results presented above correspond to this range of $\lambda$. Practically the same $\alpha^{-1}$ is also valid for UV light with $\lambda<260 \mathrm{~nm}$. Addition- ally, since silver is much less reflective at this low wavelengths, in experimental realizations larger absorbed fluences $F$ can be obtained. Optical penetration depth is a few times larger for wavelengths around $\lambda=320 \mathrm{~nm}$ due to a dip in $k$ associated with the plasma frequency of silver. However, we have checked that even in these cases the results do not change considerably.

\section{Conclusions}

In this work, we have studied effects of initial surface temperature and laser pulse properties in femtosecond laser induced desorption. As a case study system we have used $\mathrm{O}_{2}$ adsorbed on $\mathrm{Ag}(110)$ which possesses four adsorption wells. In a previous work we showed that desorption is electronically induced if the molecule is adsorbed in the hollow wells, and that desorption is phonon induced if the molecule is adsorbed in the bridge wells. Here, we have explored the possibility of tuning the desorption yield ratios between different wells.

We have shown that the initial surface temperature is an important parameter that greatly affects the desorption yields. The largest ratios of the desorption yields among the four wells are obtained for low initial surface temperatures. At high initial surface temperatures desorption yields from the four wells (and the ratios of desorption yields among them) saturate to one as all molecules become desorbed. As the desorption from the hollow wells, which is electronically induced, is always higher than the desorption from bridge wells, low initial surface temperatures can be used to favour the electronically induced process.

We have also shown that the effect of changing the laser pulse width or wavelength has only a modest influence on desorption. In general, desorption from the four wells increases as $m$-th root of the laser pulse width, with $m=4-10$. However, this does not result in a significant change in the desorption yield ratios among the four wells.

\section{Acknowledgements}

I.L., M.A. and J.I.J. acknowledge the Basque Departamento de Educación, Universidades e Investigación, the University of the Basque Country UPV/EHU (Grant No. IT-756-13) and the Spanish Ministerio de Economía y Competitividad 
(Grant No. FIS2013-48286-C2-2-P). P.S. acknowledges support by the Deutsche Forschungsgemeinschaft (DFG) through project Sa 547/8. Computational resources were provided by the DIPC computing center.

\section{References}

[1] W. Ho, Femtosecond laser-induced dynamical quantum processes on solid surfaces (DQPSS), Surf. Sci. 363 (13) (1996) 166 - 178. doi:10.1016/0039-6028(96)00130-6

[2] P. Saalfrank, Quantum dynamical approach to ultrafast molecular desorption from surfaces, Chem. Rev. 106 (10) (2006) 4116-4159. doi:10.1021/cr0501691

[3] H. Guo, P. Saalfrank, T. Seideman, Theory of photoinduced surface reactions of admolecules, Prog. Surf. Sci. 62 (7-8) (1999) 239 - 303. doi:10.1016/ S0079-6816(99) 00013-1.

[4] P. Saalfrank, M. Nest, I. Andrianov, T. Klamroth, D. Kröner, S. Beyvers, Quantum dynamics of laserinduced desorption from metal and semiconductor surfaces, and related phenomena, J. Phys.: Condens. Matter 18 (30) (2006) S1425. doi:10.1088/0953-8984/18/ $30 / \mathrm{S} 05$

[5] M. Bonn, S. Funk, C. Hess, D. N. Denzler, C. Stampfl, M. Scheffler, M. Wolf, G. Ertl, Phonon- versus electronmediated desorption and oxidation of $\mathrm{CO}$ on $\mathrm{Ru}(0001)$, Science 285 (5430) (1999) 1042-1045. doi:10.1126/ science.285.5430.1042

[6] J. A. Prybyla, T. F. Heinz, J. A. Misewich, M. M. T. Loy, J. H. Glownia, Desorption induced by femtosecond laser pulses, Phys. Rev. Lett. 64 (1990) 1537-1540. doi : 10.1103/PhysRevLett.64.1537

[7] L. M. Struck, L. J. Richter, S. A. Buntin, R. R. Cavanagh, J. C. Stephenson, Femtosecond laser-induced desorption of $\mathrm{CO}$ from $\mathrm{Cu}(100)$ : Comparison of theory and experiment, Phys. Rev. Lett. 77 (1996) 4576-4579. doi:10.1103/PhysRevLett.77.4576

[8] P. Szymanski, A. L. Harris, N. Camillone, Temperaturedependent femtosecond photoinduced desorption in CO/Pd(111), J. Phys. Chem. A 111 (49) (2007) 12524. doi:10.1021/jp075923w

[9] F.-J. Kao, D. G. Busch, D. Cohen, D. Gomes da Costa, W. Ho, Femtosecond laser desorption of molecularly adsorbed oxygen from Pt(111), Phys. Rev. Lett. 71 (1993) 2094-2097. doi:10.1103/PhysRevLett.71.2094

[10] P. Szymanski, A. L. Harris, N. Camillone, Adsorptionstate-dependent subpicosecond photoinduced desorption dynamics, J. Chem. Phys. 126 (2007) 214709. doi:10.1063/1.2735594

[11] D. N. Denzler, C. Frischkorn, C. Hess, M. Wolf, G. Ertl, Electronic excitation and dynamic promotion of a surface reaction, Phys. Rev. Lett. 91 (2003) 226102. doi: 10.1103/PhysRevLett.91.226102

[12] C. Springer, M. Head-Gordon, J. C. Tully, Simulations of femtosecond laser-induced desorption of $\mathrm{CO}$ from $\mathrm{Cu}(100)$, Surf. Sci. 320 (1-2) (1994) L57-L62. doi:10.1016/0039-6028(94)00569-9

[13] C. Springer, M. Head-Gordon, Simulations of the femtosecond laser-induced desorption of $\mathrm{CO}$ from $\mathrm{Cu}(100)$ at 0.5 ML coverage, Chem. Phys. 205 (1-2) (1996) $73-$ 89. doi:10.1016/0301-0104(95) 00316-9
[14] A. C. Luntz, M. Persson, S. Wagner, C. Frischkorn, M. Wolf, Femtosecond laser induced associative desorption of $\mathrm{H} 2$ from $\mathrm{Ru}(0001)$ : Comparison of first principles theory with experiment, J. Chem. Phys. 124 (24) (2006) 244702. doi:10.1063/1.2206588

[15] G. Füchsel, T. Klamroth, S. Monturet, P. Saalfrank, Dissipative dynamics within the electronic friction approach: the femtosecond laser desorption of H2/D2 from $\mathrm{Ru}(0001)$, Phys. Chem. Chem. Phys. 13 (2011) 8659-8670. doi:10.1039/C0CP02086A

[16] G. Füchsel, J. C. Tremblay, T. Klamroth, P. Saalfrank, C. Frischkorn, Concept of a single temperature for highly nonequilibrium laser-induced hydrogen desorption from a ruthenium surface, Phys. Rev. Lett. 109 (2012) 098303. doi:10.1103/PhysRevLett.109.098303

[17] S. I. Anisimov, B. L. Kapeliovich, T. L. Perel'man, Electron emission from metal surfaces exposed to ultrashort laser pulses, JETP 39 (2) (1974) 375.

[18] I. Lončarić, M. Alducin, P. Saalfrank, J. I. Juaristi, Femtosecond-laser-driven molecular dynamics on surfaces: Photodesorption of molecular oxygen from Ag(110), Phys. Rev. B 93 (2016) 014301. doi:10.1103/ PhysRevB.93.014301

[19] S. A. Adelman, J. D. Doll, Generalized langevin equation approach for atom/solidsurface scattering: General formulation for classical scattering off harmonic solids, J. Chem. Phys. 64 (6) (1976) 2375-2388. doi: 10.1063/1.432526

[20] J. C. Tully, Dynamics of gas-surface interactions: 3D generalized langevin model applied to fcc and bcc surfaces, J. Chem. Phys. 73 (4) (1980) 1975-1985. doi: 10.1063/1.440287

[21] H. F. Busnengo, M. A. Di Césare, W. Dong, A. Salin, Surface temperature effects in dynamic trapping mediated adsorption of light molecules on metal surfaces: $\mathrm{H}_{2}$ on $\mathrm{Pd}(111)$ and $\mathrm{Pd}(110)$, Phys. Rev. B 72 (2005) 125411. doi:10.1103/PhysRevB.72.125411

[22] H. F. Busnengo, W. Dong, A. Salin, Trapping, molecular adsorption, and precursors for nonactivated chemisorption, Phys. Rev. Lett. 93 (2004) 236103. doi: 10.1103/PhysRevLett.93.236103

[23] I. Lončarić, M. Alducin, J. I. Juaristi, Dissociative dynamics of $\mathrm{O} 2$ on $\mathrm{Ag}(110)$, Phys. Chem. Chem. Phys. 17 (2015) 9436-9445. doi:10.1039/C4CP05900J

[24] J. I. Juaristi, M. Alducin, R. Díez Muiño, H. F. Busnengo, A. Salin, Role of electron-hole pair excitations in the dissociative adsorption of diatomic molecules on metal surfaces, Phys. Rev. Lett. 100 (2008) 116102. doi:10.1103/PhysRevLett.100.116102

[25] P. Echenique, R. Nieminen, R. Ritchie, Density functional calculation of stopping power of an electron gas for slow ions, Solid State Commun. 37 (10) (1981) 779 - 781. doi:10.1016/0038-1098(81) 91173-X

[26] M. Brandbyge, P. Hedegård, T. F. Heinz, J. A. Misewich, D. M. Newns, Electronically driven adsorbate excitation mechanism in femtosecond-pulse laser desorption, Phys. Rev. B 52 (1995) 6042-6056. doi: 10.1103/PhysRevB.52.6042

[27] S. Babar, J. H. Weaver, Optical constants of $\mathrm{Cu}, \mathrm{Ag}$, and Au revisited, Appl. Opt. 54 (3) (2015) 477-481. doi:10.1364/A0.54.000477

[28] M. N. Polyanskiy, Refractive index database URL http://refractiveindex.info 